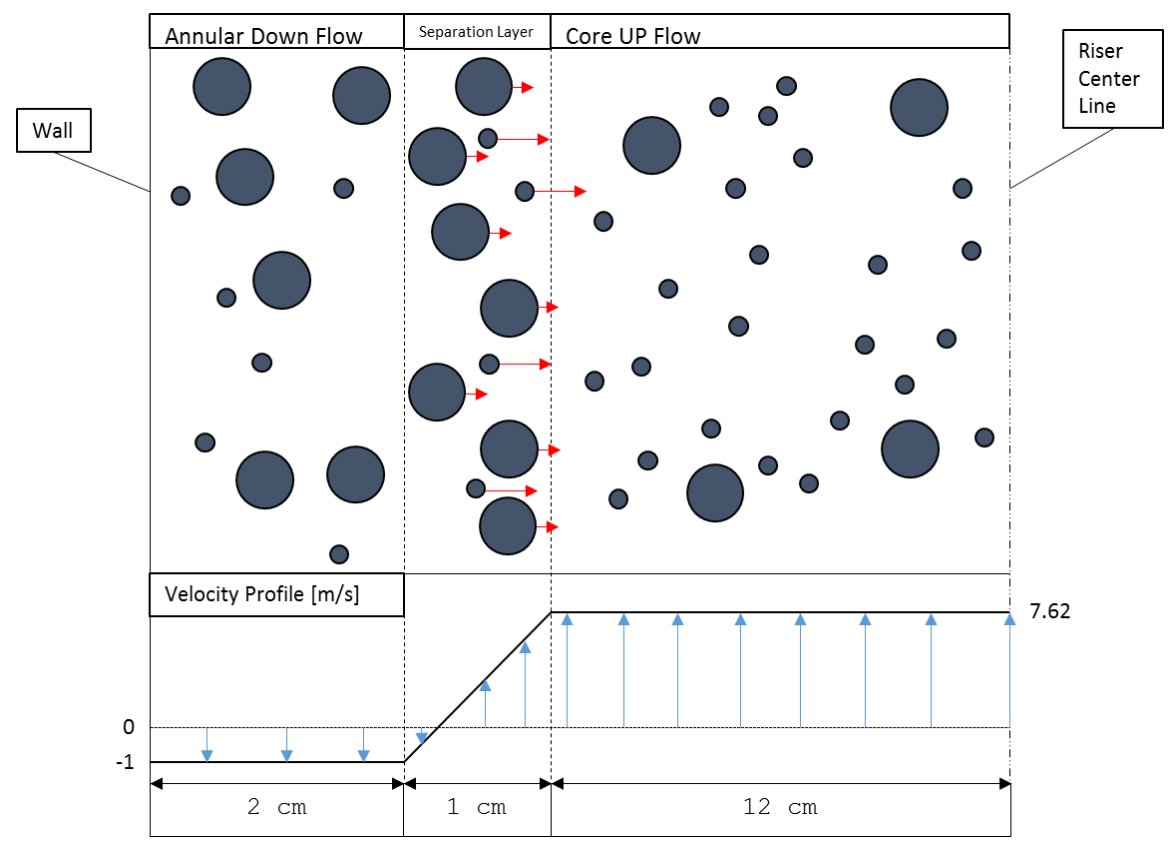

Figure 5. Caricature of Particle behavior and Velocity Profile.

[Red arrows indicate acceleration.] 


\title{
Lateral particle size segregation in a riser under core annular flow conditions due to the Saffman Lift Force
}

\author{
Ronald W. Breault ${ }^{1}$, Steven L. Rowan ${ }^{\mathrm{t}}$, Esmail Monazam ${ }^{*}$ and Kyle T. Stewart ${ }^{\mathrm{t}}$ \\ National Energy Technology Laboratory \\ United States Department of Energy \\ Morgantown, WV \\ toak Ridge Institute for Science and Education (ORISE) \\ Morgantown, WV \\ *REM Engineering Services \\ Morgantown, WV
}

\begin{abstract}
$\underline{\text { ABSTRACT }}$
It has been observed under certain flow conditions that there is a measured increase in fine particle concentration at the center of the circulating fluidized bed riser just below the exit to the cyclone. It is hypothesized that the Saffman force might be responsible for this phenomena. Therefore, this research paper discusses the likelihood of the existence of "Saffman" forces, or lift due to viscous shear, in cylindrical, circulating fluidized bed (CFB) risers operating in the core annular regime.
\end{abstract}

\footnotetext{
${ }^{1}$ Corresponding author, R. W. Breault, V:304-285-4486, F:304-285-4469, ronald.breault@ netl.doe.gov
} 


\section{INTRODUCTION}

Circulating fluidized beds (CFB's) have been widely used in a variety of industries, including the electrical generation, chemical and pharmaceutical industries, because of their high level of mixing of gas and solid phases, resulting in excellent mass and heat transfer characteristics [1-2]. The operating parameters of a CFB are largely determined based upon the specific application in question; but in general, the process involves a gas-solid matrix where the gas is the byproduct of a thermal process, such as the combustion of fossil fuels. The benefits of this process (i.e. enhanced mixing and gas-solid surface area contact) motivate the investigation of the multi-phase flow dynamics that govern the behavior of gas-solid mixtures within the riser. Due to the large solid mass flow rates associated with CFB operations, it is critical to understand and model the properties of the multi-phase fluid. Among the areas of interest to researchers are parameters that optimize absorption while permitting conditions for maximum energy generation efficiency, as well as methods and control practices that minimize the degradation of equipment and optimize the mass consumption rate of solids. However, because of the high temperatures and complex, chaotic nature of CFB hydrodynamics [2], it is not trivial to fully describe and characterize the behavior of the multi-phase gas-solid matrix.

In order to get a better understanding of riser dynamics under safer, more transparent conditions, cold flow circulating fluidized beds (CFCFBs) are often used to obtain an understanding of the particle behavior that may then be extrapolated to a full-scale industrial system. 
One area of CFB hydrodynamics that has been of interest to research is that of particle segregation within the CFB riser. This is of interest to design and process engineers due to the importance of particle size on the reaction kinetics taking place within the CFB. In general, non-homogeneous gas-particle reactions proceed at a rate that is strongly influenced by the surface area-to-volume ratio of the solid particle. The smaller the particle, the faster the chemical reactions between the particle and surrounding gas will occur. Significant particle segregation may result in sections of the CFB riser being more chemically active than other sections, possibly leading to bed instabilities.

Numerous studies have looked at segregation by particle size and/or weight axially along the CFB riser height [3-5]. In general, these studies have demonstrated a trend in which the mean particle size decreases with increasing height within the CFB riser. Other studies [6-9] have explored particle segregation in the lateral (or radial) direction within the CFB riser. These experimental studies have examined the segregation behaviors of particles that differ in either size or density. They have shown that, for a given vertical location within the CFB riser, there is a higher concentration of larger (or heavier) particles within the downward flowing annulus near the wall of the riser, and a higher concentration of smaller (or lighter) particles in the upwards flowing core region. This observed trend becomes more and more pronounced with higher vertical locations, leading to more than one investigation to observe a concentration of fines in the core of the riser near the exit [6]. A similar trend of large lateral segregation of particles hear the riser exit was observed by the authors while conducting the experiments detailed in the following section. In addition to the experimental studies, a number of models have been developed and proposed, but so far none have been able to fully capture the dynamics of this axial segregation $[3,8,10]$. 
In an effort to explain these observed trends, the authors postulate that the force responsible for the segregation is that of "lift due to viscous shear" or "Saffman force" named after P.G. Saffman who proposed its existence in 1965 [11]. In early studies by Karri and Knowlton [6], this concentration of fines in the riser core required the existence of downward flow in the annular region, and this exists in an interval of mass flux rates that is dependent upon fluid velocity; however, later studies saw evidence of this segregation present in both upwards and downwards flow in the annular region [7]. The segregation, unique to riser flow of this nature, warrants further investigation as to the physical nature of the forces present on the particles inside the riser. Therefore making the purpose of this publication be to explain and characterize the observed phenomena in order to increase the efficiency of the riser itself, as well as further develop the knowledge of particle behavior under a broader spectrum of similar flow conditions.

\section{$\underline{\text { Experimental Set-Up and Test Matrix }}$}

As previously stated, in a separate study looking at the effects of riser end geometry, the authors conducted experiments in a $15.3 \mathrm{~m}$ tall circulating fluidized bed with a bed diameter of $0.3 \mathrm{~m}$. For these experiments, a plunger was installed above the riser exhaust to allow for variation of the height of the riser top above the outlet (see Figure 1). Tests were conducted with an 80/20 mixture of $80 \mu \mathrm{m}$ and $20 \mu \mathrm{m}$ glass beads (for an average diameter of $68 \mu \mathrm{m}$ ) using superficial gas velocities of 5.12 and $7.62 \mathrm{~m} / \mathrm{s}$ and solids flux rates of 20 and $130 \mathrm{~kg} / \mathrm{m}^{2} \mathrm{~s}$. Under these operating conditions, a dense upward flow (aka pneumatic transport) regime was observed within the CFB riser for the 
lower solids flux cases, and core-annular flow conditions was observed for the higher solids flux cases.

Experiments were conducted with the plunger located at different heights above the riser outlet. For each experiment, particle velocities and average particle diameters were measured below the exit near the wall via high speed video, as well as near the riser centerline using a fiber optic probe. The data obtained from these measurements, and presented in Figures 2 and 3, show that the height of the plunger above the riser exit had little to no effect upon the measured average particle diameters. As seen in Figure 2, the average particle diameter for the lower solids circulation rate suggests little in the way of segregation, whereas there is a significant level of segregation evident in the higher solids circulation rate data. While the data presented in Figure 3 shows a little more scatter, a similar trend is evident when comparing the average particle diameter and bed voidage. These results are in agreement with the earlier observations of Karri and Knowlton [6].

As shown in Figure 2, when the CFB is operating within the core-annular flow regime (as evidenced by the higher solids flux rates), the measured average particle diameter near the centerline of the riser was $55 \mu \mathrm{m}$. This implied the presence of a mixture consisting of $58.33 \%$ particles of $80 \mu \mathrm{m}$ and $41.66 \%$ particles of $20 \mu \mathrm{m}$ diameters within the core, as opposed to the initial 80/20 mixture. However, the measured average particle size near the wall indicated that the mixture properties in the annulus remained $80 / 20$ of $80 \mu \mathrm{m}$ and $20 \mu \mathrm{m}$ particles, respectively. Moreover, measurements of the average particle density across the entire cross-sectional area revealed a constant overall particle density of the same proportion as the annular down flow region. From 
these observations, it can be concluded that if the average 80/20 particle mixture of the entire bed is maintained within the down-flowing annular region, and the upwards-flowing core region is characterized by a higher percentage of small particles and lower percentage of large particles, then mass conservation suggests that there must be a third, intermediate, region that is characterized by a much higher percentage of large particles. As a consequence, the authors present the particle density caricature shown in Figure 4. It is the authors' belief that this "separation layer" is the result of small particle migration towards the riser core brought about by the viscous sheer lift force proposed by P.G. Saffman [11] as an explanation for earlier observations of the migration of small particles away from the walls in tube and capillary flows.

\section{$\underline{\text { Analysis and Discussion }}$}

As previously mentioned, the authors propose that the observed axial segregation of particles in core annular flow is the result of the for lift force due to viscous shear proposed by P. G. Saffman [11], given by,

$$
F_{s}=\frac{K \mu V a^{2} \kappa^{1 / 2}}{v^{1 / 2}}
$$

And modified by Fan et al. [2],

$$
F_{s}=\frac{K \mu}{4}\left|U-U_{p}\right| d_{p}^{2} \sqrt{\frac{1}{v}\left|\frac{\partial\left(U-U_{p}\right)}{\partial r}\right|}
$$


Where $K=6.46$ (a dimensionless constant based on numerical integration), $a$ and $d$ are the particle radius and diameter, $\mu$ and $v$ are the kinematic and dynamic fluid viscosities, $U$ and $U_{p}$ are the local gas and particle velocity vectors, $\mathrm{V}$ is the relative velocity between the particle and fluid, and $\kappa$ is the velocity gradient. The primary difference between the two equations is that Saffman utilizes a local coordinate system based at the center of the particle in his derivation, and this local origin travels with the particle, so the relative velocity difference and the velocity gradient are with respect to the particle; however, Fan utilizes a more Eulerian approach in which the gas and particle velocities are represented as vectors in a global coordinate system.

Additionally, Saffman lists three Reynolds numbers that are based upon the gas velocity gradient, relative velocity between the particle and gas, and the rotational velocity of the particle. The Saffman formulation assumes these to be less than one. In order to verify the appropriateness of applying the Saffman force, the authors have verified that the resulting Reynolds numbers are suitable in the cases being discussed.

It is easily seen from equation 2 that the resulting force is dependent upon both the difference between the local gas and particle velocities (i.e. slip velocity) and the rate of change of this velocity difference with respect to the riser radius. If the Reynolds Number is sufficiently high to produce turbulent pipe flow conditions, then an air velocity profile similar to that shown in Figure 5 can be assumed. This then leads to the assumption of a narrow separation region between the core and annular regions in which the derivative of the velocity profile is non-zero. Thus, it is the only region in which the Saffman force is non-zero, and $\frac{\partial\left(U-U_{p}\right)}{\partial r}$ can be easily approximated. 
In order to demonstrate the potential effect of the Saffman force, an idealized linear velocity profile such as the one shown in Figure 5 is assumed in order to simplify the calculations involved. With this assumption, the resulting shear in the separation layer becomes a constant value, thus simplifying the calculations of the Saffman force that follow. In addition, an average relative, or slip, velocity between the particles and gas flow is assumed. It should be noted that the wall/boundary friction effects are of lesser importance than the presence of the downward flowing particles within the annulus region responsible for the shear. While it is true that the formation of an initial boundary layer due to the no slip condition (with respect to gas velocity) at the walls will lead to a slowing of the gas flow near the walls, the very presence of the dense region of falling particles near the wall will entrain gas, resulting in a negative, non-zero net gas velocity near the wall. It is the shear region between this downward velocity and the upwards flowing core that is of primary interest in the formulation of the Saffman force.

Figure 6 shows resulting Saffman force values, as calculated via equation 2. As expected, it is seen that the magnitude of the Saffman force increases with increasing particle diameter due to the Saffman force being proportional to the particle diameter squared.

Once the force is known, the particle acceleration can be determined from Newton's Second Law,

$$
a_{p}=\frac{F_{s}}{M_{p}}
$$

Where

$$
M_{p}=\rho_{p} V_{p}=\rho_{p} \frac{\pi}{6} d^{3}
$$


Then the particle acceleration is given by,

$$
\begin{aligned}
a_{p} & =\frac{K \mu}{4 \rho_{p} V_{p}}\left|U-U_{p}\right| d_{p}^{2} \sqrt{\frac{1}{v}\left|\frac{\partial\left(U-U_{p}\right)}{\partial r}\right|} \\
& =\frac{3 K \mu}{2 \pi d_{p} \rho_{p}}\left|U-U_{p}\right| \sqrt{\frac{1}{v}\left|\frac{\partial\left(U-U_{p}\right)}{\partial r}\right|}
\end{aligned}
$$

Which has the units of $\mathrm{m} / \mathrm{s}^{2}$.

Figure 7 shows the relationship between particle acceleration and particle radius, obtained from equations 4 and 5 using the force values shown in Figure 6.

These results indicate that although larger particles are subject to higher forces due to viscous shear, the smaller particles experience a greater acceleration.

Integrating the acceleration twice and assuming the initial lateral velocity of the particle is zero, it can be shown that the time required for a particle to cross the separation layer between the annulus and core, $t_{\text {sep }}$, is given by:

$$
t_{\text {sep }}=\sqrt{\frac{2 \Delta r_{\text {sep }}}{a_{p}}}
$$

This is, of course, assuming no other forces are acting upon the particle that would hinder its lateral motion, and no particle-to-particle collisions occur. This assumption is based upon the following 
factors. First, the relative diluteness of particles in the core as compared to the annular region. Second, the radial components of particle velocity within the core are orders of magnitude less than the vertical direction. Therefore, the effects of particle-particle collisions within the core region will be comparatively negligible. It is more likely for particle-particle collisions to occur within the annulus as a result of particles rebounding off the walls and colliding with nearby particles. When this type of collision happens in the annulus, the resulting trajectories of the particles may be directed back towards the wall or inwards towards the transition region, depending upon the respective collision angles of the particles.

Furthermore, by substituting equation 5 into equation $6, t_{\text {sep }}$ becomes:

$$
t_{\text {sep }}=\sqrt{\frac{4 \Delta r_{\text {sep }} \pi d_{p} \rho_{p}}{3 K \mu\left|U-U_{p}\right| \sqrt{\frac{1}{v}\left|\frac{\partial\left(U-U_{p}\right)}{\partial r}\right|}}}
$$

Figure 8 shows the time spent in the separation layer, as a function of the particle diameter. As expected due to their higher accelerations, the smaller particles take significantly less time than the larger particles to cross the separation layer.

Finally, the lateral distance traveled per meter of vertical distance travelled in the absence of any other force or drag effects, or particle-to-particle collisions, can be calculated. To do so, first take the inverse of the gas velocity in order to obtain the time required by the particle to travel one meter in the vertical direction (assuming no slip between the particle and surrounding gas), and then 
multiply by the lateral velocity calculated from the first integral of the acceleration (over the corresponding separation layer time). The resulting lateral distance traveled is shown in Figure 9.

$$
\Delta r_{\text {lat }}=v_{p_{-} \text {lat }} t_{\text {vertical }}
$$

Figures 6-9 show that, under the idealized conditions depicted in Figure 5, increasing the particle size leads to larger resulting Saffman forces; however, the reverse trend is seen when comparing lateral particle accelerations to particle size. In other words, even though the resulting Saffman force is small for small particles, the acceleration associated with that force is actually larger for small particles. This ultimately results in greater lateral motion for small particles than for larger ones.

The numerical results presented are based upon a simplification of the flow physics involved. This simplification was implemented primarily for ease of calculation. A more rigorous examination would require a more thorough examination and knowledge of local particle and gas velocities, as well as gas velocity gradients, and measuring these experimentally can prove to be problematic. It is the opinion of the authors that, while incorporation of actual local relative velocity values for different particle sizes would lead to more accurate calculated values for the Saffman force (and the resulting lateral acceleration), the general trends would remain the same as those presented. For example, one would expect that the relative velocity between the gas and a larger particle would be larger than for a smaller particle. Using a larger relative velocity in equations 1 or 2 would result in a larger magnitude Saffman Force, but the authors expect it would have only a marginal effect on 
the resulting accelerations; as these are seen to be asymptotic with respect to particle size in Figures 7, 12-14.

\section{Comparison to Experimental Data}

The general trends depicted in Figures 6-9 can be extended to actual experimental data. In the previous Figures, a somewhat arbitrary separation layer thickness was assumed. Additionally, the average gas velocity within the core was assumed to be equal to the superficial gas velocity. However, before equations 2-8 can be applied to actual experimental conditions, it is necessary to know the actual width of the annulus, separation layer, and core region. In addition, the gas velocity within the core will be higher than the superficial gas velocity as a result of mass conservation considerations, and must be corrected.

Zenz [12] provides an expression for estimating the width of particle reflux region thickness in concurrent upflow reactors for a given superficial gas velocity, solids flux rate and solids density. For our purposes, it is assumed that this reflux region spans both the annulus and separation layer, as depicted in Figures 4 and 5. Figure 10 shows how the estimated reflux region for the higher solids flux condition provided in Figures 2 and 3 varies with superficial gas velocity for a given solids flux rate. To further demonstrate this, Figure 11 shows experimentally measured particle velocities at different radial positions for a similar solids flux rate at the same superficial gas velocity. Overlaid on top of this is a line that corresponds to the estimated reflux region thickness, normalized by the riser radius, as calculated by using the expression presented by Zenz. Figure 11 shows good agreement between the experiments and predictions. Furthermore, it can be deduced 
that the separation layer shown in Figure 5 spans the distance between the green line and the zero particle velocity point at $\mathrm{r} / \mathrm{R}=0.1$. This then leads to the conclusion that the width of the separation layer is equal to $(0.25-0.1) / 0.25=0.6$, or approximately $60 \%$ of the estimated reflux region thickness.

Chew et al. [7] studied particle segregation of binary mixtures and a continuous particle size distribution (psd) of group B particles in riser flow. The observed trends similar to those discussed earlier in this paper. Specifically, they reported decreased volume percentages of large particles near the centerline of the riser, while the volume percentage of large particles near the wall remained nearly identical to an initial distribution of 50\% large particles and 50\% small particles.

In an effort to explain the data presented in Figure 3, as well as the radial segregation results reported by Chew et al., the calculations presented in Figures 6-9 were repeated using appropriately corrected core gas velocities and estimated separation layer thicknesses based upon the estimated particle reflux region thickness as proposed by Zenz. The results of this are shown in Figures 12-14.

For each case, it is clearly shown that smaller particles will, in the absence of any collisions or other prohibitive forces, undergo a significantly larger lateral migration from the separation layer inwards into the core region. This greater inward motion of small particles will lead to a reduction in the ratio of large-to-small particles within the core, thus decreasing the average particle diameter in that portion of the riser, as seen experimentally. Other possible causes for this phenomena, namely 
electrostatic forces and particle reflections from the roof, have been considered and rejected for the following reasons. Numerous configurations leading to particle reflections off the riser top have been examined without successfully arriving at a mechanistic path that would concentrate fines in the center of the riser as has been measured. The analysis shows that the direction of the reflective bounce is not a function of the particle size; but only of the approach angle. Therefore, the measured concentration of the fines in the riser core cannot be due to reflective bounce. The fines concentration at the core are also not caused from electrostatic effects. In the presence of strong electrostatics, there tends to be a deposition of the smaller particle sizes on the walls. This is the opposite effect from what is being reported and therefore, does not explain the fines concentration in the core. Furthermore, an anti-static compound (Larostat) was added to the $15 \mathrm{~m}$ riser, as well as air humidification, in order to decrease the level of tribo-electric charging. In summary then, the Saffman force provides a plausible physical reason for the particle segregation phenomena that has been previously observed.

\section{CONCLUSION}

Axial and radial particle segregation in circulating fluidized bed risers has been studied extensively using both experimental and computational means. Axial segregation has most commonly been attributed to the interactions between the fluid flow and the terminal settling velocity of the particles. However, the cause of radial segregation of particles in core annular flows has been a largely unexplained phenomena. The authors propose that the lifting force imposed due to viscous shear, or the Saffman force, may be the driving force for this observed radial segregation. It was shown that while, for a given particle density, larger radius particles experience a larger Saffman 
force; particles of small radius are subjected to higher lateral accelerations when exposed to the viscous shear within the separation layer located between the annular and core regions within the CFB riser. These larger accelerations allow the smaller particles to travel further in the lateral direction for a given time interval than the larger particles. In essence, the separation layer acts as a sort of momentum boundary across which the larger particles are less likely to cross, while the smaller particles are readily able to cross over into the core region. As a consequence, the percentage of smaller particles found in the core is significantly higher, and the larger particles are more concentrated closer to the riser walls; as is seen in experimental results. Clearly more experimentation and data are required to solidify these claims, but the data and calculations suggest that the Saffman force can, if not completely, then at least partially explain this phenomena.

\section{ACKNOWLEDGEMENTS}

This research was supported in part by an appointment to the National Energy Technology Laboratory Research Participation Program, sponsored by the U.S. Department of Energy and administered by the Oak Ridge Institute for Science and Education.

\section{DISCLAIMER}

The U.S. Department of Energy, NETL, ORISE and REM contributions to this paper were prepared as an account of work sponsored by an agency of the United States Government. Neither the United States Government nor any agency thereof, nor any of their employees, makes any warranty, express or implied, or assumes any legal liability or responsibility for the accuracy, completeness, or 
usefulness of any information, apparatus, product, or process disclosed, or represents that its use would not infringe privately owned rights. Reference herein to any specific commercial product, process, or service by trade name, trademark, manufacturer, or otherwise does not necessarily constitute or imply its endorsement, recommendation, or favoring by the United States Government or any agency thereof. The views and opinions of authors expressed herein do not necessarily state or reflect those of the United States Government or any agency thereof.

The authors declare no competing financial interest.

\section{REFERENCES}

[1]. D. Kunii and O. Levenspiel, Fluidization Engineering, 2nd Edition, Boston: ButterworthHeinemann, 1991.

[2]. L.-S. Fan and C. Zhu, Principles of Gas-Solid Flows, Cambridge: Cambridge University Press, 1998.

[3]. L. Huilin and D. Didaspow, "Hydrodynamics of binary fluidization in a riser: CFD simulation using two granular temperatures," Chemical Engineering Science, vol. 58, pp. 3777-3792, 2003.

[4]. S. N. Sahu, A. K. Sahu and S. K. Biswal, "Study on mixing and segregation behaviors in particulate fluidized bed system for mineral processing," International Journal of Mining Science and Technology, vol. 25, pp. 459-464, 2015. 
[5]. Q. Wang, W. Yin, B. Zhao, H. Yang, J. Lu and L. Wei, "The segregation behaviors of fine coal particles in a coal beneficiation fluidized bed," Fuel Processing Technology, vol. 124, pp. 28-34, 2014.

[6]. S. B. Reddy Karri and T. M. Knowlton, "Flow Direction and Size Segregation of Annulus Solids in a Riser," in FLUIDIZATION IX: Proceedings of the Ninth Engineering Foundation Conference on Fluidization, Durango, CO, 1998.

[7]. J. W. Chew, R. Hays, J. G. Findlay, S. B. Reddy Karri and T. M. Knowlton, "Species segregation of binary mixtures and a continuous size distribution of Group B particles in riser flow," Chemical Engineering Science, vol. 66, pp. 4595-4604, 2011.

[8]. V. Mathiesen, t. Solberg and B. H. Hjertager, "An experimental and computational study of multiphase flow behavior in a circulating fluidized bed," International Journal of Multiphase Flow, vol. 26, pp. 387-419, 2000.

[9]. M. Das, B. C. Meikap and R. K. Saha, "Characterstics of axial and radial segregation of single and mixed particle system based on terminal settling velocity in the riser of a circulating fluidized bed," Chemical Engineering Journal, vol. 145, pp. 32-43, 2008.

[10]. R. Fan and R. O. Fox, "Segregation in polydisperse fluidized beds:Validation of a multi-fluid model," Chemical Engineering Science, vol. 63, pp. 272-285, 2008.

[11]. P. G. Saffman, "The lift on a small sphere in slow shear flow," J. Fluid. Mech., vol. 22, pp. 385-400, 1965.

[12]. F. Zenz, "Predicting the degree of particle refluxing in cocurrent upflow risers," Powder Technology, vol. 97, pp. 146-150, 1997. 
Figure(s)

Figure 1. Schematic of $\mathrm{CFB}$ with the loop seal solids return loop and T-shape riser exit with Insert depicting adjustable baffle in the blind-T exit fully extended to the top of the crossover $(\mathrm{h} / \Phi=0)$.

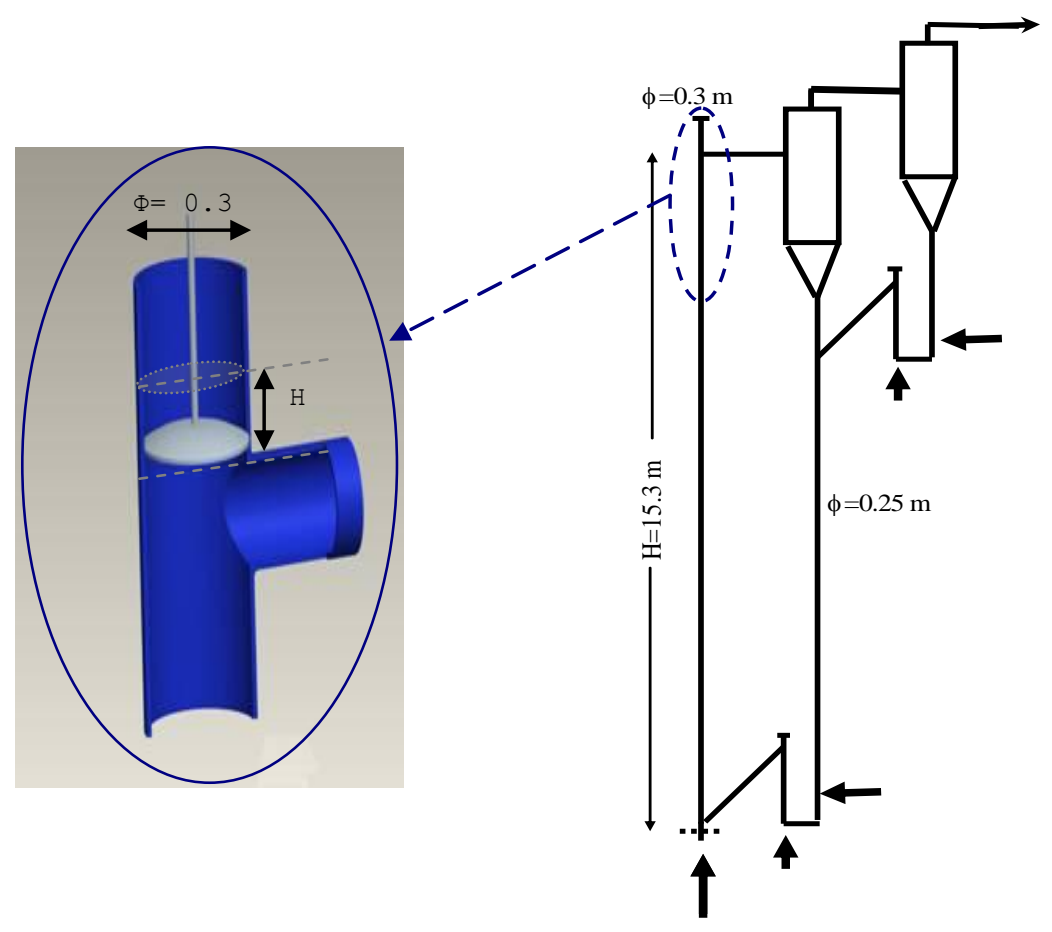


Figure 2. Particle diameter vs. plunger height

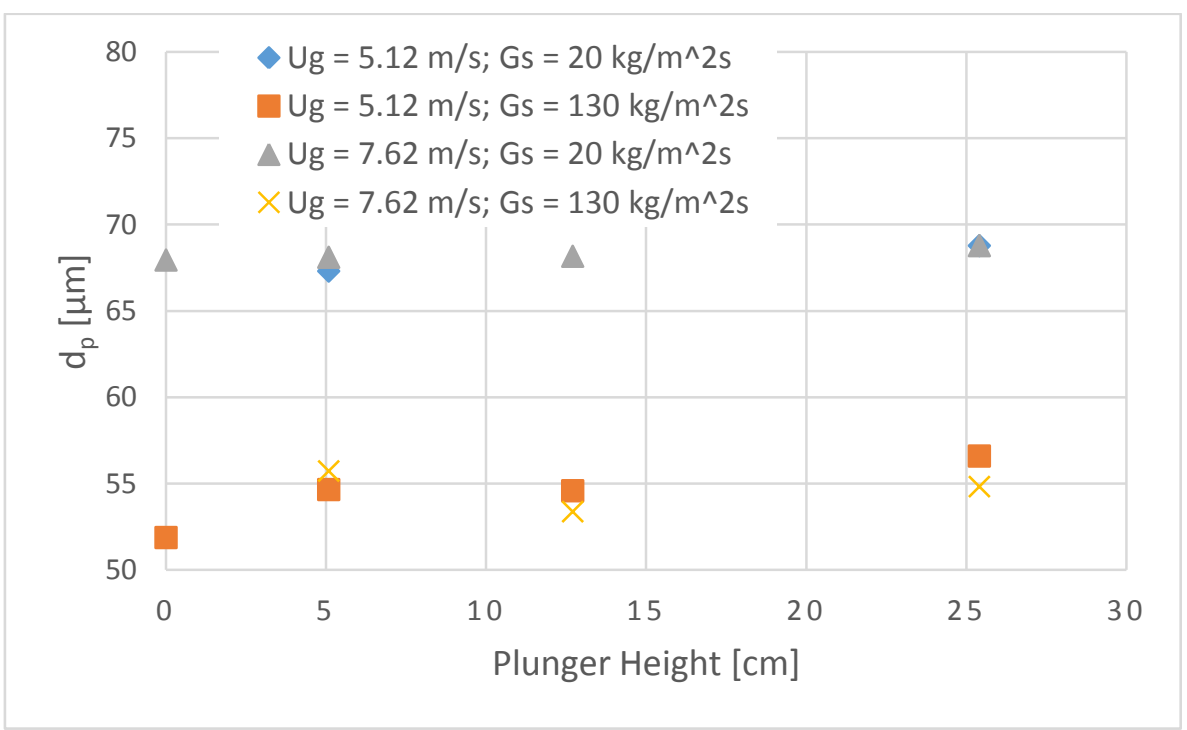


Figure 3. Particle diameter vs. cross-sectional voidage

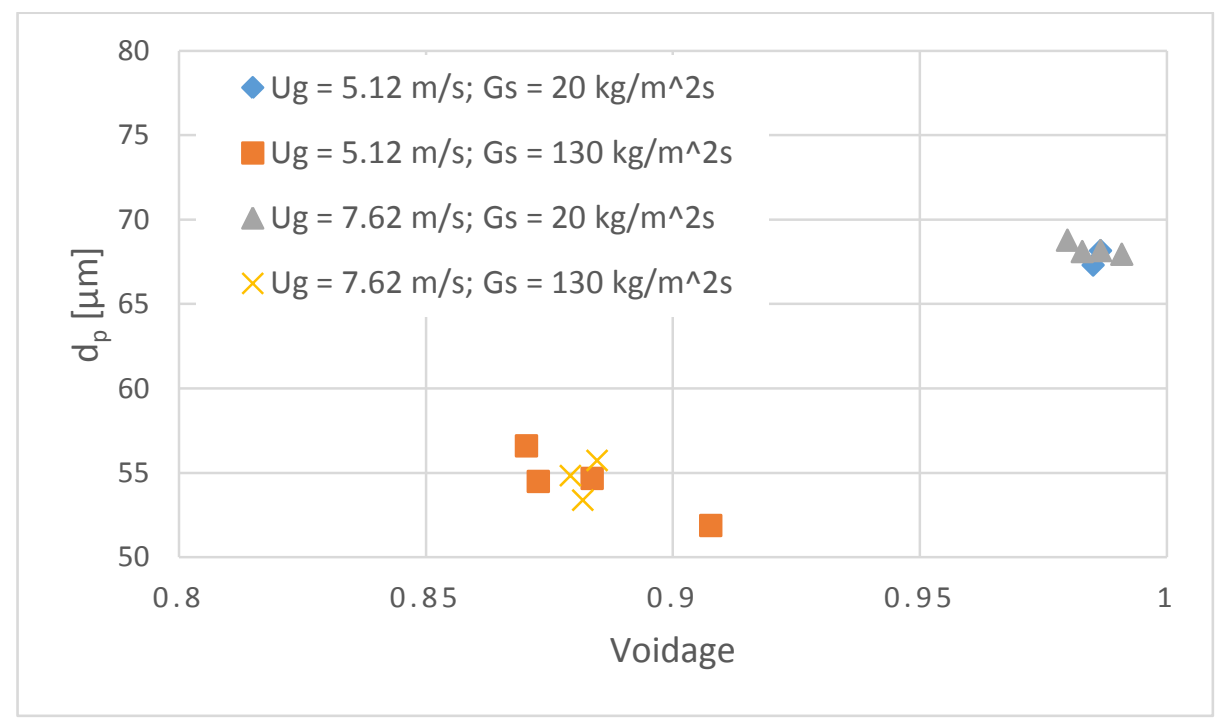


Figure 4. Radial distribution of theoretical average particle diameter.

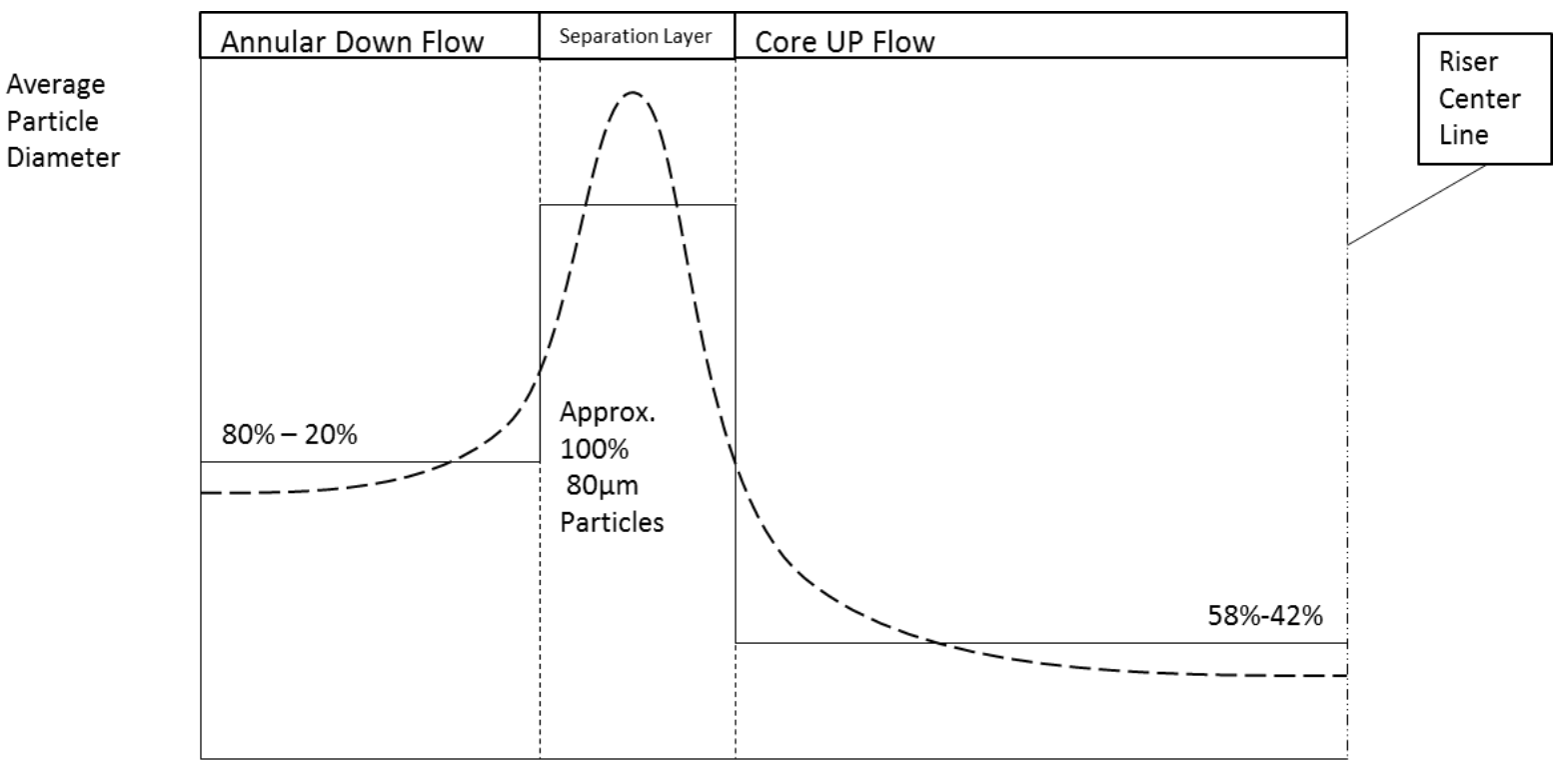


Figure 5. Caricature of Particle behavior and Velocity Profile. [Red arrows indicate acceleration.]

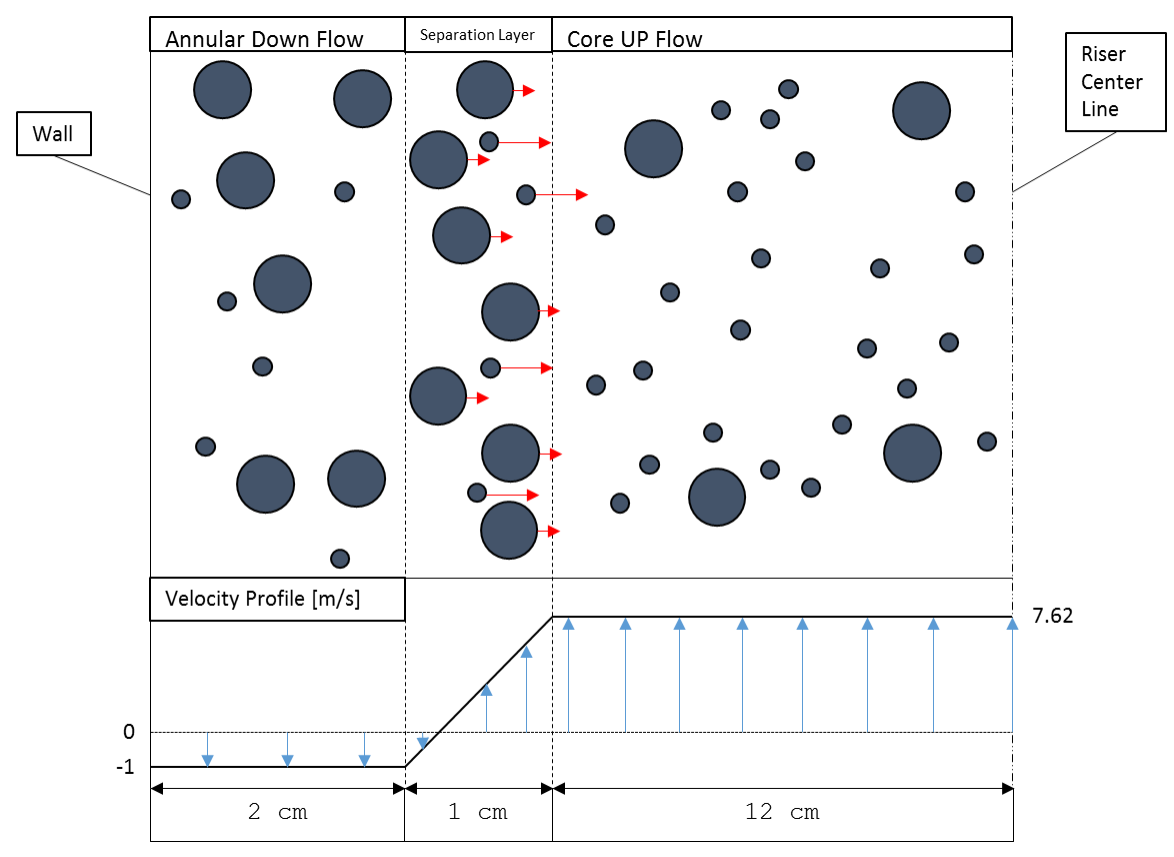


Figure 6. Saffman force vs. particle diameter

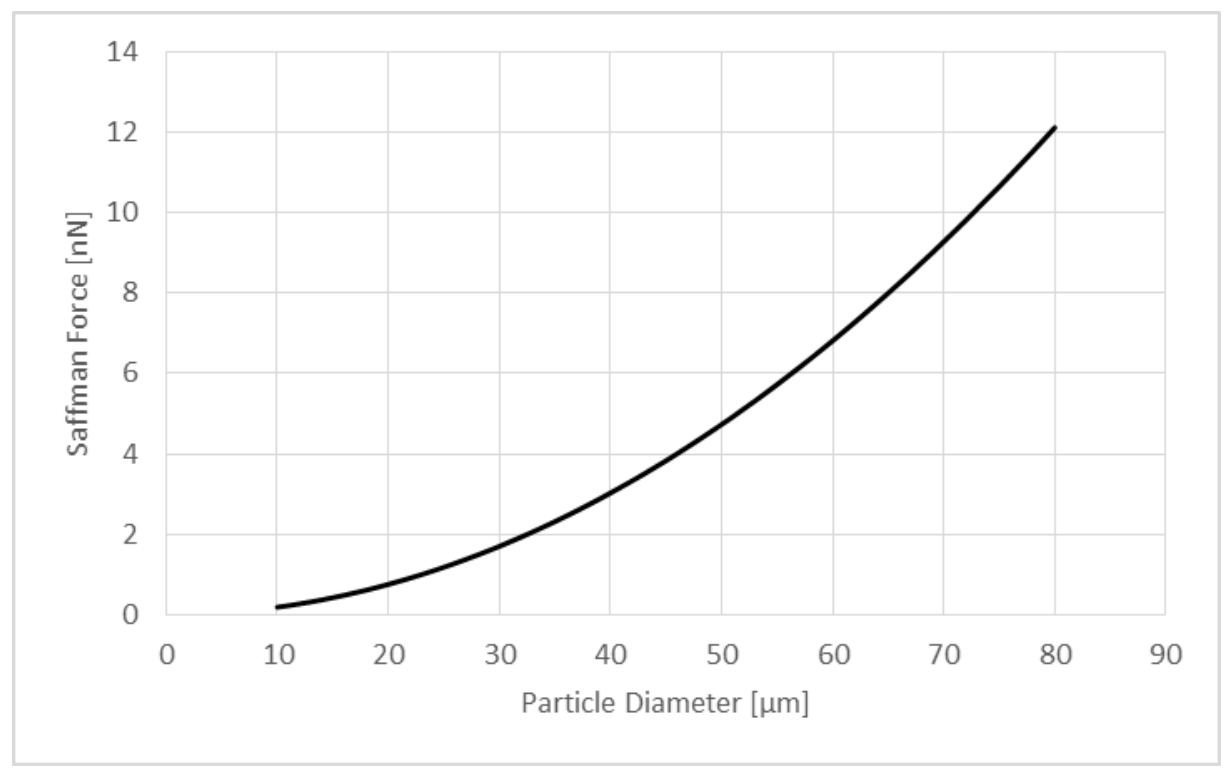


Figure 7. Particle acceleration vs. particle radius

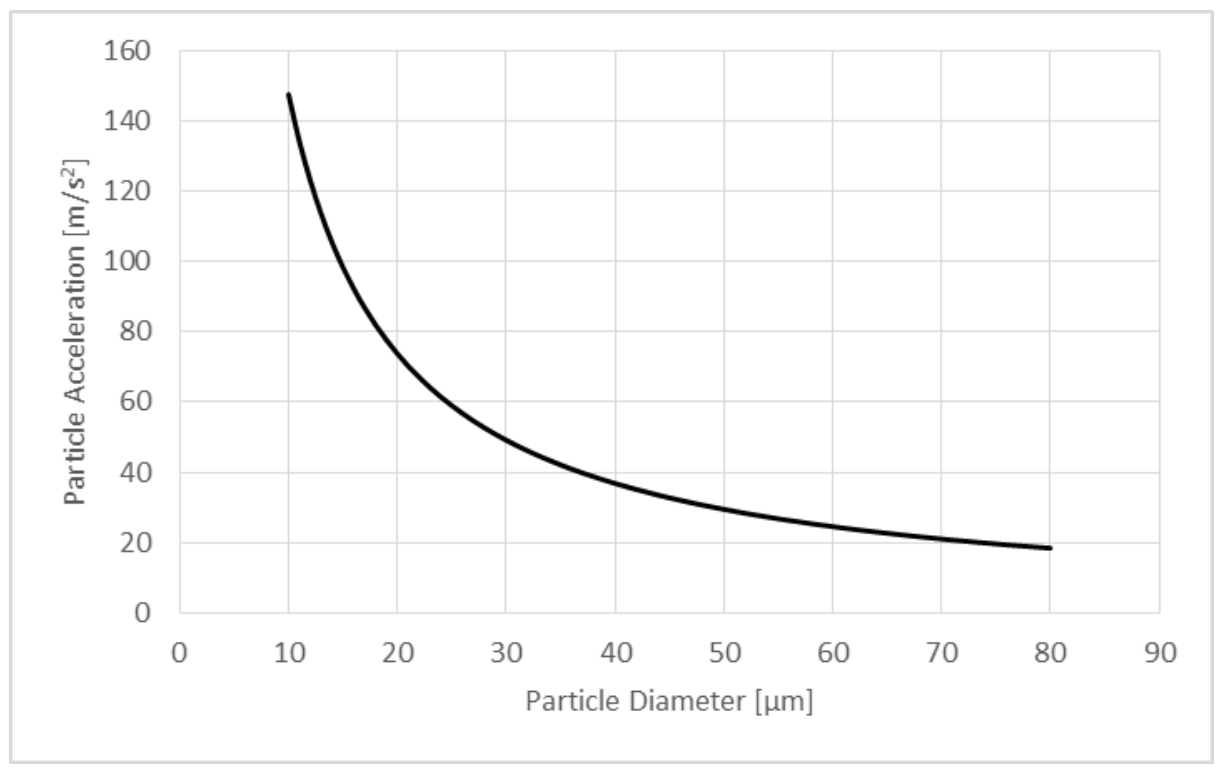


Figure 8. Separation layer time vs. particle diameter.

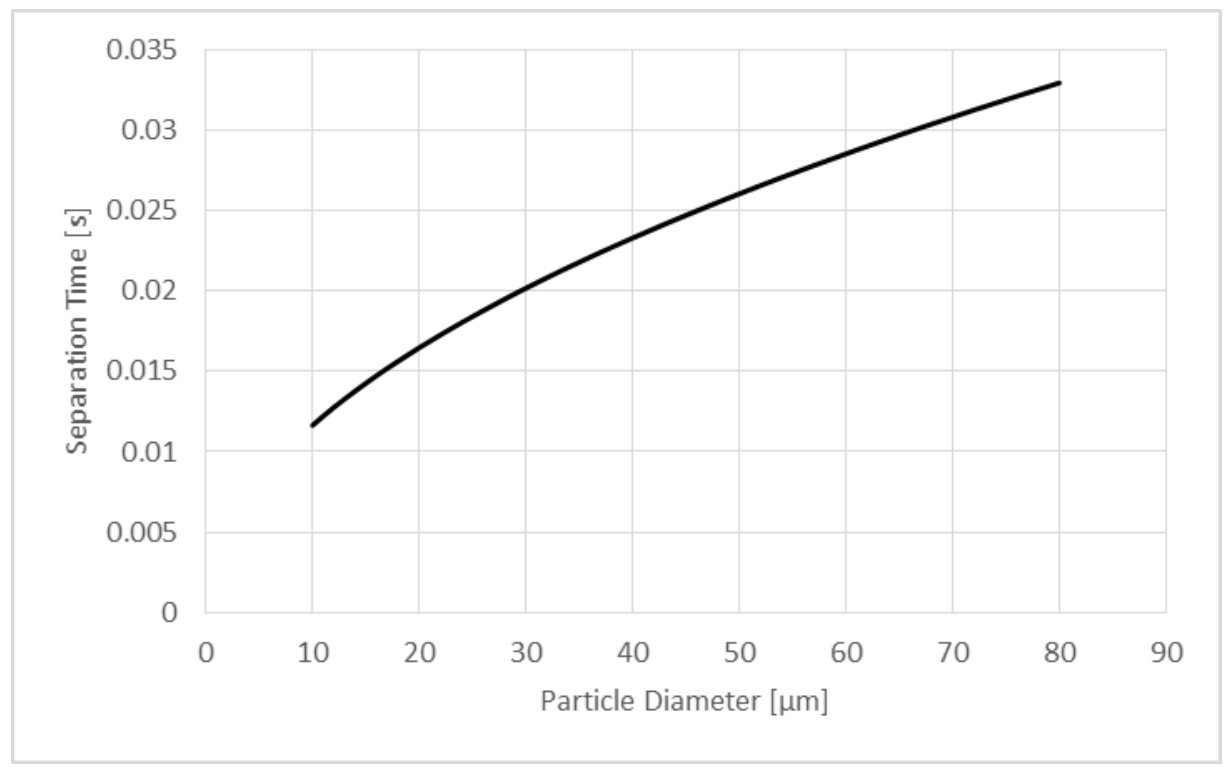


Figure 9. Particle lateral distance traveled per meter of vertical travel vs. particle diameter

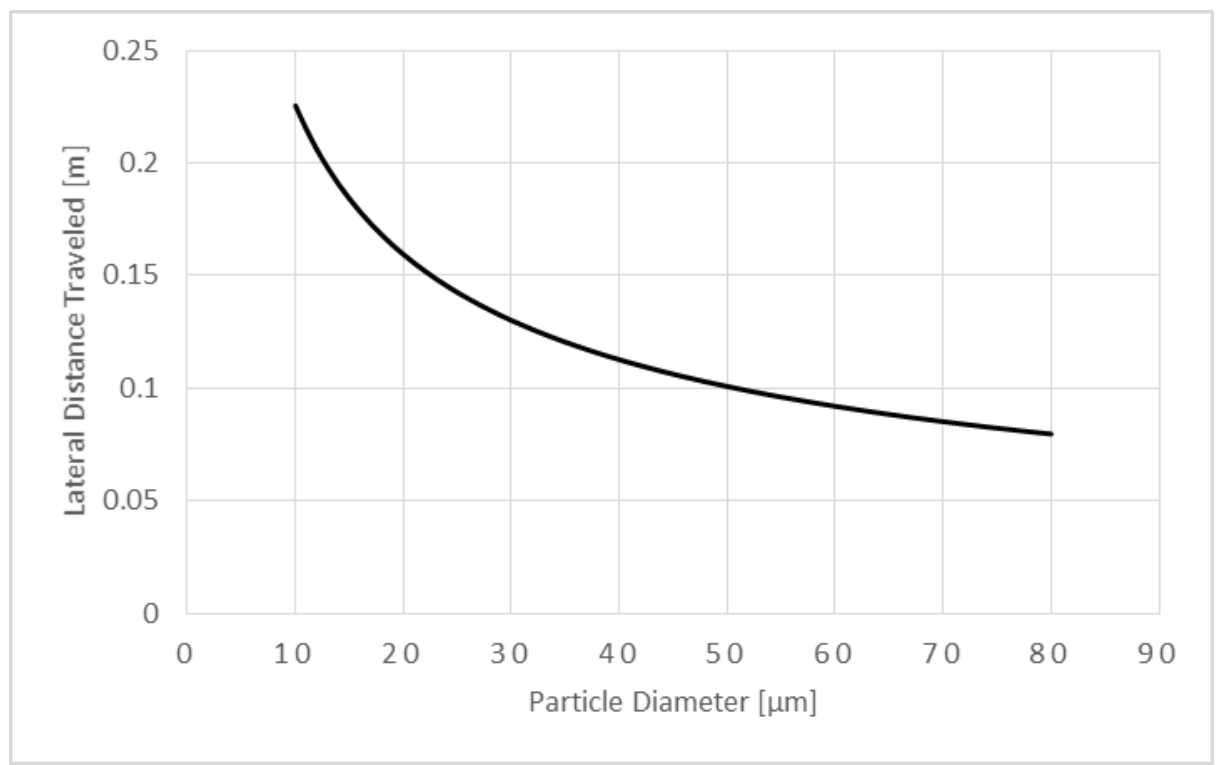


Figure 10: Annulus, or reflux region, thickness vs superficial gas velocity for solids flux rate $=130 \mathrm{~kg} / \mathrm{m}^{2} \mathrm{~s}$.

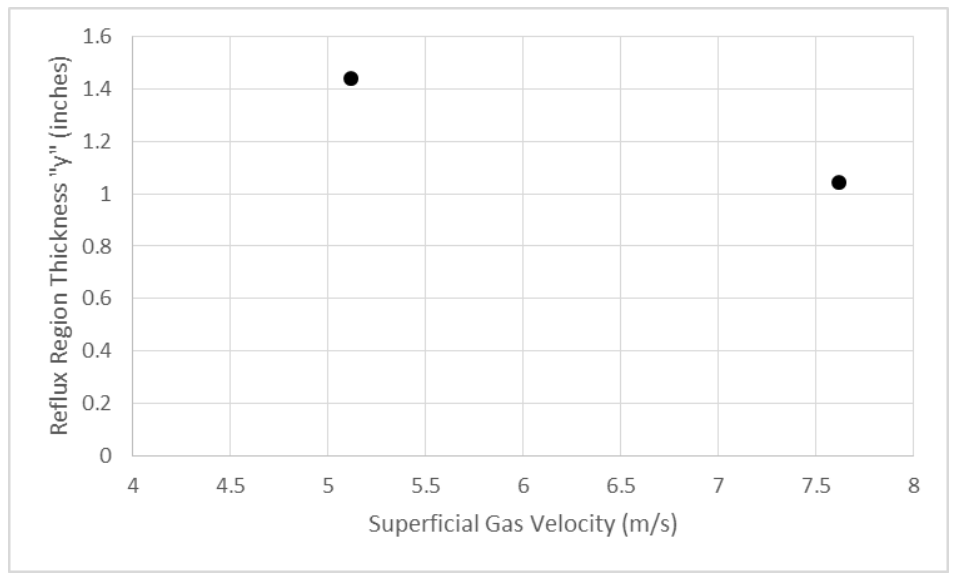


Figure 11: Measured particle velocity for different normalized radial positions superimposed with predicted annulus region thickness using relationship from Zenz [12].

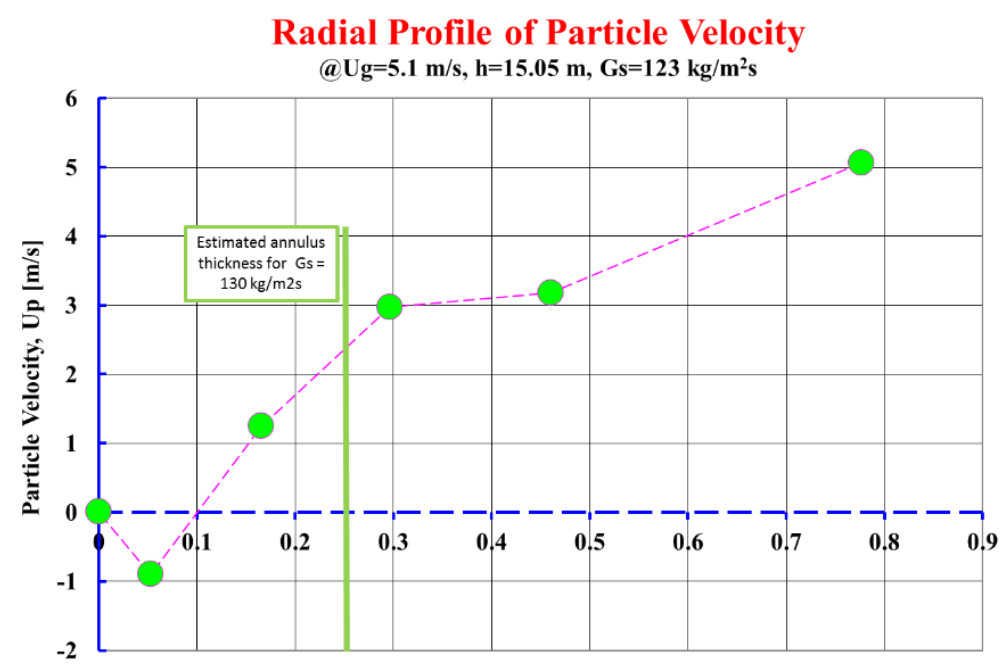

Dimensionless Radius from Wall, r/R [-] 
Figure 12: Effects of particle size on Saffman force, particle lateral acceleration, separation layer time, and lateral distance travelled per meter vertical distance travelled for solid flux rate $=130 \mathrm{~kg} / \mathrm{m}^{2} \mathrm{~s}$, and superficial gas velocity $=5.12$ and $7.62 \mathrm{~m} / \mathrm{s}$.
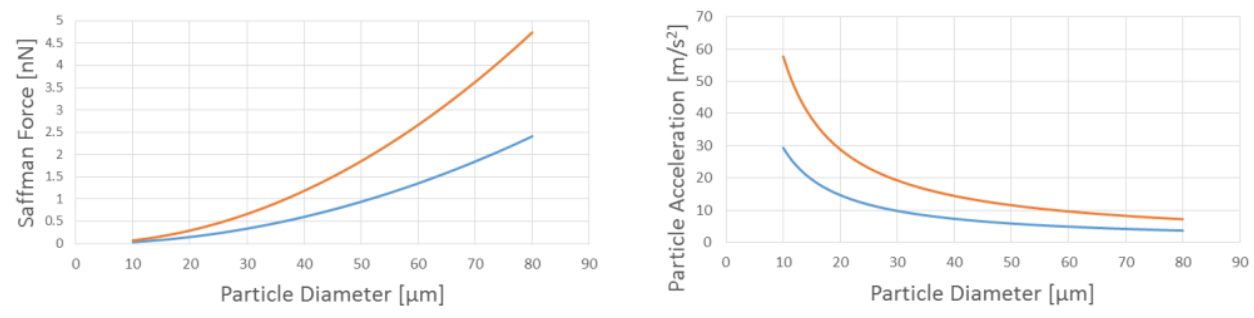

$-U g=5.12-U g=7.62$

$-U g=5.12-U g=7.62$
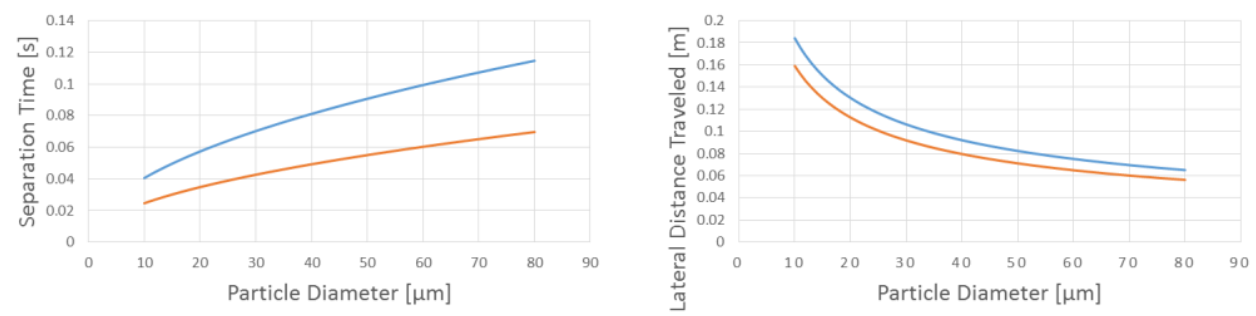

$-U g=5.12-U g=7.62$

$-U g=5.12-U g=7.62$ 
Figure 13: Effects of particle size on Saffman force, particle lateral acceleration, separation layer time, and lateral distance travelled per meter vertical distance travelled for size difference binary mixture (see Figure 4, Chew et al. [7]). Solid flux rate $=120 \mathrm{~kg} / \mathrm{m}^{2} \mathrm{~s}$, Superficial gas velocity $=13.5$ and $17 \mathrm{~m} / \mathrm{s}$.
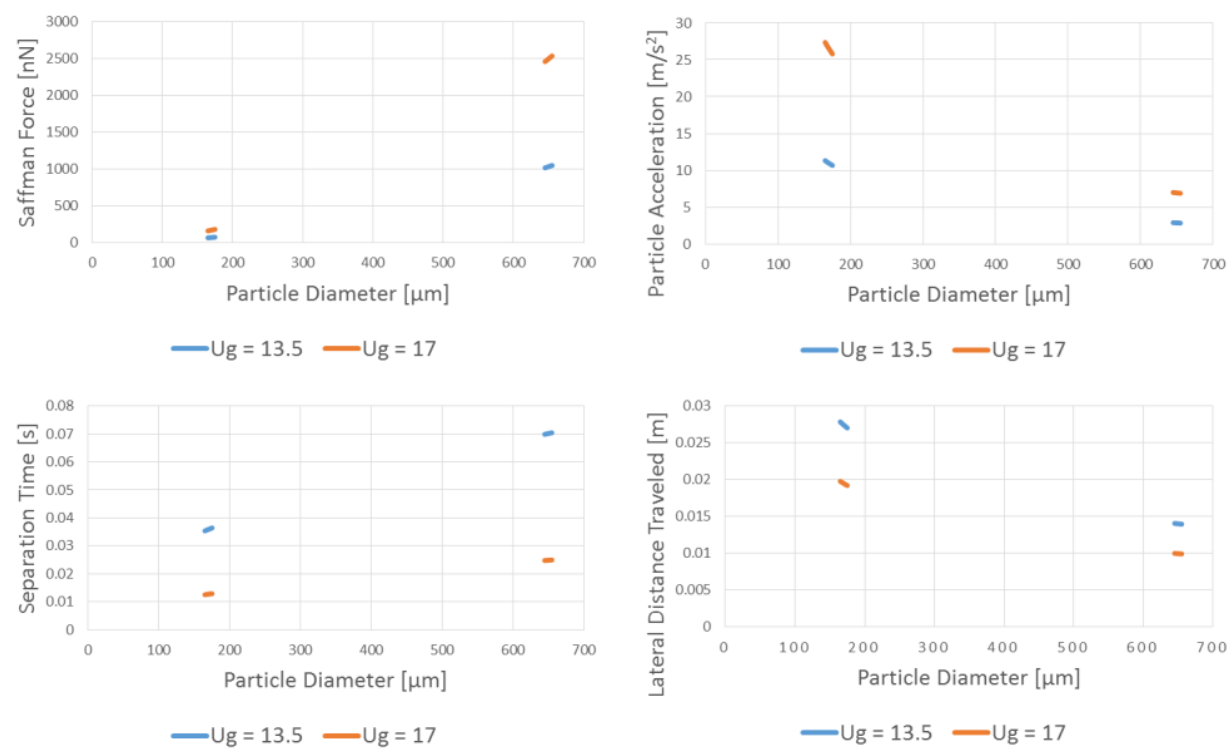
Figure 14: Effects of particle size on Saffman force, particle lateral acceleration, separation layer time, and lateral distance travelled per meter vertical distance travelled for size continuous PSD small glass mixture (see Figure 6, Chew et al. [7]). Solid flux rate $=50 \mathrm{~kg} / \mathrm{m}^{2} \mathrm{~s}$, Superficial gas velocity $=10$ and $15 \mathrm{~m} / \mathrm{s}$.
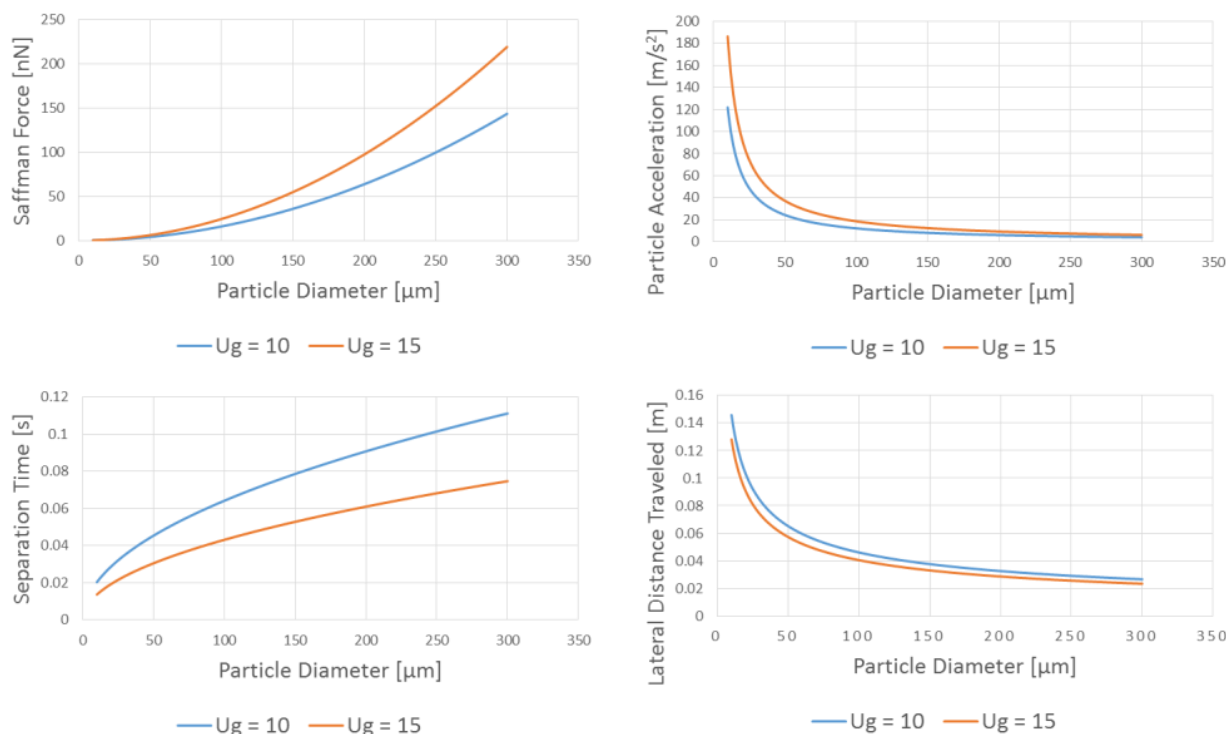